\title{
Osteoporosis: Current Concepts
}

\author{
Ibrahim Akkawi ${ }^{1}$ Hassan Zmerly ${ }^{1}$ \\ 1 Orthopaedics and Traumatology Unit, Villa Erbosa Hospital, \\ Bologna, Italy \\ Joints 2018;6:122-127.
}

\begin{abstract}
Address for correspondence Ibrahim Akkawi, MD, Orthopaedics and Traumatology Unit, Villa Erbosa Hospital, Bologna 40129, Italy (e-mail: i.akkawi@libero.it).
\end{abstract}

\begin{abstract}
Keywords

- osteoporosis

- DEXA

- bone mineral density

- anabolic agents

- antiresorptive agents
\end{abstract}

Osteoporosis is a worldwide disease characterized by reduction of bone mass and alteration of bone architecture resulting in increased bone fragility and increased fracture risk. Causes of osteoporosis include increasing age, female sex, postmenopausal status, hypogonadism or premature ovarian failure, low body mass index, ethnic background, rheumatoid arthritis, low bone mineral density (BMD), vitamin D deficiency, low calcium intake, hyperkyphosis, current smoking, alcohol abuse, immobilization, and long-term use of certain medications. The diagnosis of osteoporosis is established by measurement of BMD of the hip and spine using dual energy X-ray absorptiometry. According to the World Health Organization criteria, osteoporosis is defined as a BMD that lies 2.5 standard deviation or more below the average value for young healthy women. Bone turnover biomarker detection may be useful in monitoring osteoporosis treatment and assessing fracture risk but not for diagnosis of osteoporosis. Management of osteoporosis consists of nonpharmacological interventions, which are recommended for all subjects, and pharmacological therapy in all postmenopausal women who have had an osteoporotic fracture or have BMD values consistent with osteoporosis.

\section{Introduction}

Osteoporosis is a worldwide disease characterized by reduction of bone mass and alteration of bone architecture resulting in increased bone fragility and increased fracture risk. ${ }^{1-4}$ The prevalence of osteoporosis is expected to increase significantly in the future because of aging of the population. 5,6 Osteoporosis mainly occurs in postmenopausal women and elderly men. ${ }^{7}$

Approximately 200 million people suffer from osteoporosis and approximately 8.9 million fractures are caused by osteoporotic fracture. ${ }^{6}$ These fractures occur mainly at the hip, vertebrae, and distal forearm ${ }^{7}$ and are associated with significant morbidity, mortality, and reduced quality of life, attributed not only to the fracture itself but also to the high prevalence of comorbidities in this population of patients. ${ }^{6,7}$ Moreover, osteoporosis represents a major concern of the health care systems because of its growing economic burden. ${ }^{5}$ In the United States, costs related to osteoporosis fractures were estimated at $\$ 13.8$ billion. $^{8}$

received

September 3, 2017

accepted

May 2, 2018

published online

June 14, 2018
DOI https://doi.org/

10.1055/s-0038-1660790. ISSN 2282-4324.

\section{Definition}

According to the National Institutes of Health Consensus Development Panel on Osteoporosis, ${ }^{9}$ osteoporosis is defined as "a skeletal disorder characterized by compromised bone strength leading to an increased risk of fracture." Moreover, according to the World Health Organization (WHO) criteria, osteoporosis is defined as a bone mineral density (BMD) that lies 2.5 standard deviation (SD) or more below the average value for young healthy women (a T-score of $<-2.5 \mathrm{SD}$ ). ${ }^{10}$

Osteoporosis can be subdivided into primary osteoporosis, which includes postmenopausal osteoporosis (type I) and senile osteoporosis (type II), and secondary osteoporosis, which has a clearly definable etiologic mechanism such as malabsorption, medications such as glucocorticoids, and some diseases such as hyperparathyroidism. ${ }^{11,12}$

\section{Risk Factors}

Causes of osteoporosis include increasing age, female sex, postmenopausal status, hypogonadism or premature

Copyright $\odot 2018$ Georg Thieme Verlag License terms KG Stuttgart · New York 
ovarian failure, low body mass index, ethnic background (white persons are at higher risk than black persons), rheumatoid arthritis (RA), low BMD, vitamin D deficiency, low calcium intake, hyperkyphosis, current smoking, alcohol abuse, immobilization, and long-term use of certain medications, such as glucocorticoids, anticoagulants, anticonvulsants, aromatase inhibitors, cancer chemotherapeutic drugs, and gonadotropin-releasing hormone agonists. ${ }^{2,4,13,14}$

\section{Diagnosis}

Osteoporosis is a silent disease without obvious symptoms and evidence until a fracture occurs. ${ }^{5}$ Thus, screening by dual energy X-ray absorptiometry (DEXA) is important to obtain an early diagnosis and to avoid fractures. ${ }^{5}$ All women aged 65 years or older and men aged 70 years or older, postmenopausal women with medical causes of bone loss (e.g., steroid use) regardless of age, postmenopausal women aged 50 years or older with additional risk factors for fracture (e.g., current smoker, RA, history of hip fracture in a parent), and postmenopausal women with a fragility fracture should be screened for osteoporosis by BMD measurement at the hip and lumbar spine as recommended by the U.S. Preventive Services Task Force (USPSTF), the National Osteoporosis Foundation, and by other guidelines. ${ }^{10-12,15}$

BMD is mainly described as T-score, which represents the number of SD by which the BMD in an individual differs from the mean value expected in young healthy individuals. ${ }^{6}$ Based on the report of the WHO, BMD with a T-score above $1 \mathrm{SD}$ is classified as normal BMD, a T-score between -1.0 and $-2.5 \mathrm{SD}$ is classified as osteopenia, and a T-score below -2.5 SD is defined as osteoporosis. ${ }^{10}$ According to Gourlay et al, ${ }^{16}$ rescreening intervals are 15 years for those with normal BMD (T-score $\geq-1.0$ ) or mild osteopenia (T-score $<-1.0$ and $>-1.5$ ), 5 years for those with moderate osteopenia (Tscore $<-1.5$ and $>-2.0$ ), and 1 year for those with advanced osteopenia (T-score $<-2.0$ and $>-2.5$ ).

\section{Bone Turnover Biomarkers}

Bone turnover biomarkers (BTMs) are produced from the bone remodeling process and can be measured in urine or serum. They are classified as markers of bone formation (total alkaline phosphatase [total ALP], bone-specific alkaline phosphatase [B-ALP], osteocalcin [OC], procollagen type $1 \mathrm{~N}$ terminal propeptide [P1NP], and procollagen type $1 \mathrm{C}$-terminal propeptide $[\mathrm{P} 1 \mathrm{CP}]$ ) and markers of bone resorption (hydroxyproline [HYP], deoxypyridinoline [DPD], pyridinoline, tartrate-resistant acid phosphatase 5b [TRAP 5b], carboxy-terminal cross-linked telopeptide of type 1 collagen [CTX-1], and amino-terminal cross-linked telopeptide of type 1 collagen [NTX-1]). ${ }^{5,17}$

The total ALP concentration represents the sum of ALP isoenzymes from bones, liver, and intestine. In adults with normal liver function, almost $50 \%$ of ALP activity in serum is derived from bone and is produced by osteoblasts during bone formation. Therefore, measurement of total ALP activity lacks sensitivity and specificity in osteoporosis. ${ }^{17} \mathrm{~B}$-ALP is more specific for bone and, therefore, more sensitive in detecting the small changes in bone formation seen in osteoporosis. $^{17,18}$ OC is considered a specific biomarker of osteoblast function for the evaluation of bone formation rate in osteoporosis. ${ }^{5,17}$ The concentration of P1NP and P1CP in serum reflects bone formation rate, ${ }^{17}$ as they are produced during conversion of procollagen type 1 to collagen type $1 .^{19}$ Kučukalić-Selimović et al ${ }^{19}$ found that P1NP is significantly higher in postmenopausal females with osteoporosis compared with postmenopausal females with preserved bone mass, but its low specificity does not warrant its utility in diagnosing osteoporosis.

HYP is produced from the degradation of bone collagen during bone resorption. ${ }^{17}$ Since HYP can be found in other tissues such as skin and cartilage, it is considered a nonspecific marker of bone resorption. ${ }^{5}$ Pyridinoline and DPD are released during breakdown of bone and cartilage; their concentration in urine are more sensitive and specific markers of bone resorption than urinary HYP. ${ }^{17}$ TRAP $5 b$ is normally secreted by osteoclasts and is considered a specific and high sensitive biomarker of bone resorption. ${ }^{20}$ CTX-1 and NTX- 1 are released during collagen degradation. CTX-1 is a specific and sensitive biomarker of bone resorption but is influenced by food intake, thus blood withdrawal must take place in the fasting state in the morning. ${ }^{21}$ On the contrary, urinary NTX-1 is not affected by food intake. ${ }^{17}$

According to the European guidance for the diagnosis and management of osteoporosis in postmenopausal women, the most informative ones for the investigation of osteoporosis are OC and P1NP for assessing bone formation and CT1X to assess bone resorption. ${ }^{22}$

According to the current guidelines on osteoporosis management, BTM cannot diagnose osteoporosis, but changes in BTMs may be useful in monitoring osteoporosis treatment to confirm the efficacy of treatment and treatment adherence. ${ }^{11,21}$ Furthermore, the measurement of BTMs in women with a low BMD can improve the specificity of assessment of fracture risk. ${ }^{21-24}$

\section{Treatment}

The primary goal of osteoporosis therapy is to reduce the risk of fracture. ${ }^{25}$ Treatment and prevention strategies of osteoporosis and osteoporotic fractures include fall avoidance by correcting decreased visual acuity, reducing consumption of medication that alters alertness and balance, reducing fall hazards in the home (slippery floors, obstacles, insufficient light), practicing physical activity to improve muscle strength, balance, and maintaining bone mass, the avoidance of cigarette smoking and excessive alcohol intake, and adequate dietary intake of protein, calcium, and vitamin D. ${ }^{11,12,26}$ In women, the recommended daily allowance (RDA) for calcium is $1,000 \mathrm{mg} / \mathrm{d}$ for age range of 19 to 50 years and increases to $1,200 \mathrm{mg} / \mathrm{d}$ for older than 50 years; in men, the RDA of calcium is $1,000 \mathrm{mg} / \mathrm{d}$ for age range of 19 to 70 years and increases to $1,200 \mathrm{mg} / \mathrm{d}$ for older than 70 years. The RDA for vitamin D is $600 \mathrm{IU} / \mathrm{d}$ for men and women aged 19 to 70 years and increases to $800 \mathrm{IU} / \mathrm{d}$ for those older than 
70 years. ${ }^{2,12}$ All postmenopausal women, regardless of their bone density or clinical risk factors for osteoporosis should observe these recommendations. ${ }^{12}$

The North American Menopause Society ${ }^{12}$ recommends adding osteoporosis drug therapy in all postmenopausal women who have had an osteoporotic vertebral or hip fracture, all those who have BMD values consistent with osteoporosis at the lumbar spine, femoral neck, or total hip region and all those who have $\mathrm{T}$-scores from -1.0 to -2.5 and a 10 -year risk of major osteoporotic fracture of at least $20 \%$ or a risk of hip fracture of at least $3 \%$.

Pharmacological agents are classified into two groups: those that decrease bone resorption (antiresorptive agents) and those that increase skeletal formation (anabolic agents). ${ }^{7}$

Antiresorptive drugs (bisphosphonates [BPs], denosumab, strontium ranelate, estrogen replacement therapy [ERT], and selective estrogen receptor modulator [SERM]) reduce the rate of bone resorption and the rate of bone formation. The overall changes are associated with increases of BMD, but up to a certain point due to the coupling between bone resorption and formation. ${ }^{7}$ Anabolic drugs (teriparatide, romosozumab) stimulate bone formation and partially bone resorption.

\section{Bisphosphonates}

BPs are the first option for the treatment of osteoporosis. These drugs, such as alendronate, risedronate, ibandronate, etidronate, clodronate, and zoledronic acid, are potent inhibitors of bone resorption and mainly increase the BMD of trabecular bones. ${ }^{3,26}$ It has been showed that BPs reduce vertebral, nonvertebral, and hip fractures compared with placebo in postmenopausal osteoporotic women. ${ }^{4}$

Oral BPs are associated with mild upper gastrointestinal symptoms such as dysphagia and esophagitis. ${ }^{4,12,26}$ Moreover, BPs are associated with atypical femoral fractures (AFF), and osteonecrosis of the jaw (ONJ), albeit these events are rare (5.9/100,000 person-years and 2/100,000 patient-years, respectively). ${ }^{11,27,28}$ Park-Wyllie et al ${ }^{29}$ found that among older women, treatment with a BP for more than 5 years was associated with an increased risk of AFF. Galis et $\mathrm{al}^{30}$ observed that the risk of ONJ development is increased by several factors, such as duration of BP therapy, administration route of BP, type of BP, invasive dental procedures or dental prostheses, oncological disease, Caucasian origin, and multiple myeloma. Other adverse effects include hypocalcemia, influenza-like symptoms, uveitis, and episcleritis. ${ }^{4}$ BPs are contraindicated in patients with low serum calcium and severe renal impairment (creatinine clearance below 30-35 $\mathrm{mL})^{12}$

After 3 years of treatment with intravenous zoledronic acid or 5 years with oral BP, a treatment break should be considered as suggested by the American Society for Bone and Mineral Research, USPSTF, and the American College of Physicians (ACP), whereas if there are characteristics indicative of high fracture risk, such as old age, low hip T-score or high fracture risk score, previous major osteoporotic fractures, or fractures on therapy, it is recommended to continue the treatment with BPs up to 10 years. ${ }^{4,11}$

\section{Denosumab}

Receptor activator of nuclear factor-kappa B ligand (RANKL) is expressed on the membrane of osteoblastic cells and binds to its receptor, RANK, on the surface of osteoclasts, which is essential for osteoclast formation, activity, and survival. Denosumab is a human monoclonal antibody that binds RANKL and prevents it from combining with RANK on the osteoclast membrane, thus inhibiting its action, favoring bone formation over bone resorption, and increasing bone mass and strength in both trabecular and cortical bone, thereby reducing radiographic vertebral, nonvertebral, and hip fractures in postmenopausal osteoporotic women. 4,31,32

Similarly to BP, denosumab therapy is associated with mild upper gastrointestinal symptoms, AFF and ONJ; moreover, it is associated with increased risk for infection, rash, and/or eczema. ${ }^{4}$ Therefore, in patients considered at low risk of fracture after 5 years of treatment, discontinuation of denosumab is recommended, whereas in patients with a low BMD or multiple vertebral fractures or a high fracture risk score, it is advisable to continue treatment with denosumab for up to 10 years and consolidate with a single infusion of zoledronic acid. ${ }^{32}$

\section{Strontium Ranelate}

Strontium ranelate (Sr RAN) increases the osteoblast differentiation while osteoclast formation is inhibited simultaneously, thus Sr RAN significantly improves bone mass and quality, and increases bone strength; therefore, it remarkably reduces the risk of vertebral, nonvertebral, and hip fractures in a wide range of postmenopausal women with documented osteoporosis. $^{33}$

The most common reported adverse effects of Sr RAN are cardiovascular events, venous thromboembolism, myocardial infarction, gastrointestinal discomfort, and signs and symptoms of nervous system such as headache, seizure, and memory loss. ${ }^{34}$ Sr RAN is not recommended for patients with severe renal impairment (creatinine clearance below $30 \mathrm{~mL} / \mathrm{min}$ ), patients with a previous history of thrombophlebitis, and patients with established current or previous history of ischemic heart disease, peripheral arterial disease and/or cerebrovascular disease, and uncontrolled hypertension. ${ }^{11,26}$

The Italian guidelines for the diagnosis, prevention, and management of osteoporosis stated that the use of Sr RAN must be restricted to the treatment of severe osteoporosis in postmenopausal women or in adult men with high risk of fracture for whom treatment with other medicinal products approved for the treatment of osteoporosis is not possible. ${ }^{11}$

\section{Estrogen Replacement Therapy}

Since menopause is characterized by estrogen deficiency that results in bone loss, it has been suggested that the use of ERT is effective for prevention of osteoporosis in postmenopausal women, but it is not recommended as the firstline preventive treatment of osteoporosis. ${ }^{13}$ ERT is associated with an increased risk of coronary heart disease, breast cancer, stroke, and dementia. ${ }^{11,26}$ 


\section{Selective Estrogen Receptor Modulator}

Raloxifene is the only SERM widely available for the prevention and treatment of postmenopausal osteoporosis. ${ }^{26}$ It reduces the risk of vertebral fractures in postmenopausal women with osteoporosis but has no effect on the risk for nonvertebral fractures. Therefore, it is not a first-line agent for postmenopausal osteoporosis. Moreover, the long-term use of raloxifene increases the risk of venous thromboembolic events. $^{2}$

ACP recommends against using menopausal estrogen therapy or menopausal estrogen plus progestogen therapy or raloxifene for the treatment of osteoporosis in women. ${ }^{4}$

\section{Calcitonin}

Calcitonin inhibits osteoclastic bone resorption. ${ }^{26}$ The use of a nasal spray formulation of salmon calcitonin to treat osteoporosis is associated with an increased risk of cancer. Hence, salmon calcitonin has been withdrawn from the market in Europe and Canada. Although it is available in the United States for treating osteoporosis (but not for prevention), the Food and Drug Administration Advisory Committee has not recommended it. ${ }^{12,35}$ Drug-related adverse effects include nausea, local inflammation, and flushing of the face or hands when calcitonin is given as an injection, and local nasal irritation with the nasal spray formulation. $^{12}$

\section{Teriparatide}

Teriparatide is a peptide corresponding to the $34 \mathrm{~N}$-terminal amino acids of parathyroid hormone (PTH). Candidates for teriparatide treatment include patients with contraindications to oral and intravenous BPs, those who have had a major osteoporotic fracture while receiving oral BPs, and treatment-naive persons with very low BMD T-scores $(\leq-3.5) .^{2}$ It is contraindicated in conditions characterized by abnormally increased bone turnover (e.g., preexisting hypercalcemia, metabolic bone diseases other than primary osteoporosis, unexplained elevation of ALP, severe renal impairment, prior skeletal irradiation, or patients with skeletal malignancies or bone metastasis). ${ }^{12,26}$

Teriparatide treatment reduces vertebral and nonvertebral fractures among women with postmenopausal osteoporosis. ${ }^{4}$ Treatment with teriparatide is characterized by an early period dominated by bone formation that lasts for 6 to 9 months and is referred to as the anabolic window. Then, bone resorption increases and mitigates the overall bone anabolic effect. ${ }^{36}$

Teriparatide treatment is associated with mild upper gastrointestinal symptoms, nausea, pain in the limbs, dizziness, headache, hypercalcemia, hypercalciuria, hyperuricemia, and hypotension. ${ }^{4,26}$ Moreover, the use of teriparatide is limited to 2 years in any life span, and this is because of the development of osteosarcoma in preclinical animal studies. ${ }^{3}$

\section{Romosozumab}

Romosozumab is another anabolic drug, which is still under clinical development. It is an antibody of sclerostin that represent an important component of the Wnt signaling pathway, a well-known metabolic route to drive osteoblast proliferation. Thus, it increases bone formation, bone mass, and strength at various skeletal sites. ${ }^{7}$ Romosozumab has been evaluated in a double-blind study at a monthly dose of $210 \mathrm{mg}$ versus placebo in 7,180 postmenopausal women who had a T-score of -2.5 to -3.5 at the total hip or femoral neck. At 12 months, new vertebral fractures had occurred in 16 of 3,321 patients $(0.5 \%)$ in the romosozumab group, as compared with 59 of $3,322(1.8 \%)$ in the placebo group (representing a $73 \%$ lower risk with romosozumab). Clinical fractures had occurred in 58 of 3,589 patients (1.6\%) in the romosozumab group, as compared with 90 of 3,591 (2.5\%) in the placebo group (a $36 \%$ lower risk with romosozumab). Nonvertebral fractures had occurred in 56 of 3,589 patients (1.6\%) in the romosozumab group and in 75 of 3,591 (2.1\%) in the placebo group. ${ }^{37}$

\section{Combined Treatments}

Osteoporosis treatments are currently limited to the use of a single drug at a fixed dose, ${ }^{38}$ while combination pharmacotherapy is not recommended. ${ }^{2}$ Combination of more than two antiresorptive agents have demonstrated very limited additive effects on bone mass. ${ }^{39}$ Similarly, combination of PTH and teriparatide with BPs or SERM does not have an overall superior effect on BMD compared with monotherapy. ${ }^{40,41}$ On the contrary, it has been shown that combined therapy of teriparatide with denosumab or zoledronate may be an effective treatment option for patients who do not respond to teriparatide monotherapy. ${ }^{25}$ A study found that the addition of alendronate to teriparatide after the first 9 months of teriparatide treatment contributed to a reopening of the anabolic window and led to a return of bone resorption to levels comparable at the initiation of teriparatide therapy, whereas bone formation was less suppressed and remained elevated. ${ }^{36}$ Furthermore, the recent denosumab and teriparatide administration study indicated that the combination of denosumab and teriparatide produced a more prominent effect on increasing BMD and decreasing fracture risk than each drug did alone. ${ }^{38}$ It also indicated that teriparatide treatment after denosumab was associated with transient bone loss in lumbar spine and proximal femur and with prolonged BMD decrease in distal radius, while teriparatide followed by denosumab continuously increased the BMD of lumbar spine and proximal femur. The authors concluded that it is necessary to consider the timing of teriparatide use, as well as the order of sequential use of teriparatide, in the long-term management of patients with osteoporosis. ${ }^{38}$ Finally, since discontinuation of estrogens, SERMs, denosumab, or teriparatide therapy is associated with a rapid loss of their effects on BMD and BTMs over 1 to 2 years, a follow-up treatment with BP should be considered to reduce or prevent the rebound increase in bone turnover. $^{38}$

\section{Conclusion}

Osteoporosis is a skeletal disorder characterized by compromised bone strength leading to an increased risk of fracture. 
It is defined as a BMD that lies 2.5 SD or more below the average value for young healthy women, as measured with DEXA. According to the current guidelines on osteoporosis management, BTMs cannot diagnose osteoporosis, but changes in BTMs may be useful in monitoring osteoporosis treatment to confirm the efficacy of treatment and treatment adherence and can improve the specificity of assessment of fracture risk. All postmenopausal women should be encouraged to maintain a healthy weight; to obtain adequate calcium, vitamin $\mathrm{D}$, and protein intake; to participate in appropriate exercise; to avoid excessive alcohol consumption and smoking; and to utilize measures that prevent falls. Finally, drug therapy is recommended in all postmenopausal women who have a history of osteoporotic vertebral or hip fracture, in those who have BMD values consistent with osteoporosis, and in those who have T-scores from -1.0 to -2.5 and a 10 -year risk of major osteoporotic fracture.

\section{Conflict of Interest}

None.

\section{References}

1 Dunnewind T, Dvortsin EP, Smeets HM, et al. Economic consequences and potentially preventable costs related to osteoporosis in the Netherlands. Value Health 2017;20(06):762-768

2 Ensrud KE, Crandall CJ. Osteoporosis. Ann Intern Med 2017;167 (03):ITC17-ITC32

3 Fukumoto S, MatsumotoT. Recent advances in the management of osteoporosis. F1000 Res 2017;6:625

4 Qaseem A, Forciea MA, McLean RM, Denberg TD; Clinical Guidelines Committee of the American College of Physicians. Treatment of low bone density or osteoporosis to prevent fractures in men and women: a clinical practice guideline update from the American College of Physicians. Ann Intern Med 2017;166(11): 818-839

5 Kuo TR, Chen $\mathrm{CH}$. Bone biomarker for the clinical assessment of osteoporosis: recent developments and future perspectives. Biomark Res 2017;5:18

6 Hernlund E, Svedbom A, Ivergård M, et al. Osteoporosis in the European Union: medical management, epidemiology and economic burden. A report prepared in collaboration with the International Osteoporosis Foundation (IOF) and the European Federation of Pharmaceutical Industry Associations (EFPIA). Arch Osteoporos 2013;8:136

7 Minisola S, Cipriani C, Occhiuto M, Pepe J. New anabolic therapies for osteoporosis. Intern Emerg Med 2017;12(07):915-921

8 O'Neill TW, Roy DK. How many people develop fractures with what outcome? Best Pract Res Clin Rheumatol 2005;19(06): 879-895

9 Lorentzon M, Cummings SR. Osteoporosis: the evolution of a diagnosis. J Intern Med 2015;277(06):650-661

10 Cosman F, de Beur SJ, LeBoff MS, et al; National Osteoporosis Foundation. Clinician's guide to prevention and treatment of osteoporosis. Osteoporos Int 2014;25(10):2359-2381

11 Rossini M, Adami S, Bertoldo F, et al. Guidelines for the diagnosis, prevention and management of osteoporosis. Reumatismo 2016; 68(01):1-39

12 Management of osteoporosis in postmenopausal women: 2010 position statement of The North American Menopause Society. Menopause 2010;17(01):25-54, quiz 55-56

13 Ramírez J, Nieto-González JC, Curbelo Rodríguez R, Castañeda S, Carmona L. Prevalence and risk factors for osteoporosis and fractures in axial spondyloarthritis: a systematic review and meta-analysis. Semin Arthritis Rheum 2017;pii:S0049-0172(17) 30649-2

14 Tawaratsumida H, Setoguchi T, Arishima Y, et al. Risk factors for bone loss in patients with rheumatoid arthritis treated with biologic disease-modifying anti-rheumatic drugs. BMC Res Notes 2017;10(01):765

15 U.S. Preventive Services Task Force. Screening for osteoporosis: U. S. Preventive Services Task Force recommendation statement. Ann Intern Med 2011;154(05):356-364

16 Gourlay ML, Fine JP, Preisser JS, et al; Study of Osteoporotic Fractures Research Group. Bone-density testing interval and transition to osteoporosis in older women. N Engl J Med 2012; 366(03):225-233

17 Vasikaran SD. Utility of biochemical markers of bone turnover and bone mineral density in management of osteoporosis. Crit Rev Clin Lab Sci 2008;45(02):221-258

18 Kyd PA, Vooght KD, Kerkhoff F, Thomas E, Fairney A. Clinical usefulness of bone alkaline phosphatase in osteoporosis. Ann Clin Biochem 1998;35(Pt 6):717-725

19 Kučukalić-Selimović E, Valjevac A, Hadžović-Džuvo A. The utility of procollagen type $1 \mathrm{~N}$-terminal propeptide for the bone status assessment in postmenopausal women. Bosn J Basic Med Sci 2013;13(04):259-265

20 Nenonen A, Cheng S, Ivaska KK, et al. Serum TRACP 5b is a useful marker for monitoring alendronate treatment: comparison with other markers of bone turnover. J Bone Miner Res 2005;20(10): 1804-1812

21 Lee J, Vasikaran S. Current recommendations for laboratory testing and use of bone turnover markers in management of osteoporosis. Ann Lab Med 2012;32(02):105-112

22 Kanis JA, Burlet N, Cooper C, et al; European Society for Clinical and Economic Aspects of Osteoporosis and Osteoarthritis (ESCEO). European guidance for the diagnosis and management of osteoporosis in postmenopausal women. Osteoporos Int 2008; 19(04):399-428

23 Vasikaran S, Eastell R, Bruyère O, et al; IOF-IFCC Bone Marker Standards Working Group. Markers of bone turnover for the prediction of fracture risk and monitoring of osteoporosis treatment: a need for international reference standards. Osteoporos Int 2011;22(02):391-420

24 O'Neill S, MacLennan A, Bass S, et al; Osteoporosis Australia Medical Scientific Committee. Guidelines for the management of postmenopausal osteoporosis for GPs. Aust Fam Physician 2004;33(11):910-919

25 Kim SY, Zhang M, Bockman R. Bone mineral density response from teriparatide in patients with osteoporosis. HSS J 2017;13 (02):171-177

26 Kanis JA, McCloskey EV, Johansson H, Cooper C, Rizzoli R, Reginster JY; Scientific Advisory Board of the European Society for Clinical and Economic Aspects of Osteoporosis and Osteoarthritis (ESCEO) and the Committee of Scientific Advisors of the International Osteoporosis Foundation (IOF). European guidance for the diagnosis and management of osteoporosis in postmenopausal women. Osteoporos Int 2013;24(01):23-57

27 Feldstein AC, Black D, Perrin N, et al. Incidence and demography of femur fractures with and without atypical features. J Bone Miner Res 2012;27(05):977-986

28 Reid IR, Cornish J. Epidemiology and pathogenesis of osteonecrosis of the jaw. Nat Rev Rheumatol 2011;8(02):90-96

29 Park-Wyllie LY, Mamdani MM, Juurlink DN, et al. Bisphosphonate use and the risk of subtrochanteric or femoral shaft fractures in older women. JAMA 2011;305(08):783-789

30 Galis B, Zajko J, Hirjak D, et al. Is the prevalence of the medicationrelated osteonecrosis of the jaws underestimated, evaluation in oncological and non-oncological disease. Bratisl Lek Listy (Tlacene Vyd) 2017;118(12):724-731

31 Dubois EA, Rissmann R, Cohen AF. Denosumab. Br J Clin Pharmacol 2011;71(06):804-806 
32 Tsourdi E, Langdahl B, Cohen-Solal M, et al. Discontinuation of denosumab therapy for osteoporosis: a systematic review and position statement by ECTS. Bone 2017;105:11-17

33 Pilmane M, Salma-Ancane K, Loca D, Locs J, Berzina-Cimdina L. Strontium and strontium ranelate: historical review of some of their functions. Mater Sci Eng C 2017;78:1222-1230

34 Tabatabaei-Malazy O, Salari P, Khashayar P, Larijani B. New horizons in treatment of osteoporosis. Daru 2017;25(01):2

35 Komm BS, Morgenstern D, A Yamamoto L, Jenkins SN. The safety and tolerability profile of therapies for the prevention and treatment of osteoporosis in postmenopausal women. Expert Rev Clin Pharmacol 2015;8(06):769-784

36 Muschitz C, Kocijan R, Fahrleitner-Pammer A, Lung S, Resch H. Antiresorptives overlapping ongoing teriparatide treatment result in additional increases in bone mineral density. J Bone Miner Res 2013;28(01):196-205
37 Cosman F, Crittenden DB, Adachi JD, et al. Romosozumab treatment in postmenopausal women with osteoporosis. N Engl J Med 2016;375(16):1532-1543

38 Leder BZ, Tsai JN, Uihlein AV, et al. Two years of denosumab and teriparatide administration in postmenopausal women with osteoporosis (The DATA Extension Study): a randomized controlled trial. J Clin Endocrinol Metab 2014;99(05):1694-1700

39 Compston J. The use of combination therapy in the treatment of postmenopausal osteoporosis. Endocrine 2012;41(01):11-18

40 Cosman F, Wermers RA, Recknor C, et al. Effects of teriparatide in postmenopausal women with osteoporosis on prior alendronate or raloxifene: differences between stopping and continuing the antiresorptive agent. J Clin Endocrinol Metab 2009;94(10):3772-3780

41 Finkelstein JS, Wyland JJ, Lee H, Neer RM. Effects of teriparatide, alendronate, or both in women with postmenopausal osteoporosis. J Clin Endocrinol Metab 2010;95(04):1838-1845 